\title{
CSFIR is a Prognostic Biomarker and Correlated with Immune Cell Infiltration in the Gastric Cancer Microenvironment
}

This article was published in the following Dove Press journal:

Pharmacogenomics and Personalized Medicine

\section{Di Chen \\ Lina Xiong \\ Li Zhang \\ Honglu Yu \\ Yushuang $\mathrm{Xu}$ \\ Mengmeng Wang \\ Xin Jiang \\ Zhifan Xiong}

Department of Gastroenterology, Liyuan Hospital, Tongji Medical College, Huazhong University of Science and Technology, Wuhan, People's Republic of China
Correspondence: Zhifan Xiong Department of Gastroenterology, Liyuan Hospital, Tongii Medical College,

Huazhong University of Science and

Technology, Wuhan, 43006I, People's

Republic of China

$\mathrm{Tel}+86$ I3517281937

Email xiongzhifan@I26.com
Purpose: The tumor microenvironment (TME) plays a crucial role in the progression and prognosis of gastric cancer (GC). This study investigated TME-associated genes and explored their roles in the $\mathrm{GC}$ microenvironment.

Methods: A total of $330 \mathrm{GC}$ samples were extracted from TCGA. ESTIMATE and CIBERSORT algorithms were utilized to evaluate the stromal and immune scores of GC samples and the fraction of 22 immune cells infiltrated in the TME. Then, the TME-related differentially expressed genes (DEGs) were determined through integrative analysis. Protein-protein interaction (PPI) network and Cox regression analysis were conducted to analyze DEGs, and CSF1R was determined as the most crucial gene. We further probed the role of CSF1R in the GC microenvironment and evaluated the prognostic value of CSF1R.

Results: We identified 560 TME-related DEGs and found CSF1R associated with the development and prognosis of GC. Further analysis showed that CSF1R was involved in immune-related signaling pathways. Furthermore, CIBERSORT analysis revealed that CSF1R expression correlated with several kinds of infiltrating immune cells, including tumor-associated macrophages (TAMs), B cells, NK cells, neutrophils, eosinophils, T cells, dendritic cells, and so on.

Conclusion: In summary, CSF1R might take part in the modulation of immune-active status in the GC microenvironment and could be a promising biomarker for GC therapy and prognosis.

Keywords: CSF1R, gastric cancer, tumor microenvironment, infiltrating immune cells, ESTIMATE algorithm, CIBERSORT algorithm

\section{Introduction}

Gastric cancer (GC) is the fifth most commonly diagnosed cancer and the third leading cause of cancer mortality worldwide. ${ }^{1}$ As patients at the early stage of GC are usually asymptomatic, most of them are diagnosed with advanced stages. ${ }^{2,3}$ Although progress has been made in treatments, including surgery, chemotherapy, molecular targeted therapy, and radiotherapy, the prognosis of advanced GC patients remains pessimistic. ${ }^{3}$ Therefore, it is urgently needed to improve GC diagnosis, treatment, and prognosis.

The tumor microenvironment (TME) has recently attracted more and more attention from the cancer research community. A growing body of evidence has elucidated that the TME could influence gene expression of tumor tissues and is 
gradually recognized as a critical determinant in tumor development and therapeutic responses. ${ }^{4-6}$ The TME refers to the environment where tumor cells originate and develop. In addition to tumor cells, the TME also consists of stromal cells, the tumor vascular system, immune cells, the extracellular matrix (ECM), as well as the acidic and hypoxic environment of the tumor. ${ }^{7-9}$ Stromal cells and immune cells, as two major nontumor cell types in the TME, are considered to be of great value in tumor diagnosis and prognosis. ${ }^{10-13}$ Thus, an overall understanding of the TME may provide crucial vision into GC initiation and progression, and contribute to the development of promising diagnostic and prognostic biomarkers.

With the advent of the biological big data era, bioinformatics technique provides a more convenient platform for researchers to guide basic experiments. ${ }^{14}$ ESTIMATE was developed to evaluate stromal and immune scores for reflecting the level of stromal and immune cells infiltrated in the TME according to the transcriptional profiles of tumor tissues. ${ }^{15}$ This algorithm has been applied to colon cancer and glioblastoma, demonstrating the effectiveness of the bigdata based algorithm. ${ }^{16-18}$ Besides, CIBERSORT algorithm was performed to evaluate the fraction of 22 infiltrating immune cells in the TME using the LM22 signature. ${ }^{19}$ CIBERSORT algorithm also has been validated and applied to analyze the association between immune cell landscapes and treatment outcome in lung and breast cancer. ${ }^{20,21}$ In this study, the ESTIMATE and CIBERSORT algorithms were utilized to calculate the stromal and immune scores of GC cases and the proportion of 22 immune cells infiltrated in TME based on The Cancer Genome Atlas (TCGA) database. Furthermore, we explored the TME-related differentially expressed genes (DEGs) and found CSF1R correlated with the prognosis of GC and immune cells infiltrated in TME. We hypothesized that CSF1R might play an essential role in the GC microenvironment and could be a promising therapeutic target and prognostic biomarker.

\section{Materials and Methods}

\section{Data Preparation}

A flowchart of the study is shown in Figure 1. Gene expression data of GC samples were downloaded from the TCGA database. The corresponding patients' clinical information, including survival time, survival status, grade, and tumor stage, were also obtained from the TCGA database. Then, the ESTIMATE algorithm was applied to calculate the stromal, immune, and
ESTIMATE scores for GC samples. The acquirement and application of all data involved in this study were performed following TCGA publication guidelines and data access policies.

\section{Clinicopathological Characteristics Analysis and Survival Analysis}

Based on the stromal, immune, and ESTIMATE scores, GC patients were classified into two groups: high-score group and low-score group. We evaluated the association between clinicopathological characteristics and the stromal/immune/ESTIMATE scores. ${ }^{22}$ Furthermore, the Kaplan-Meier method was utilized to assess the association between the survival rate and the above scores. A Log rank test tested the association. The analyses were carried out through the $\mathrm{R}$ packages "survival" and "survminer", and $p<0.05$ was regarded as statistical significance.

\section{DEGs Identification}

Gene expression profiles were screened using the R package "limma", and DEGs between the low-score and high-score groups were identified. The criteria for determining DEGs were an absolute value of $\log _{2}$ fold change $\left(\left|\log _{2} \mathrm{FC}\right|\right)>1$ and false discovery rate (FDR) $<0.05$. Heatmaps of DEGs were drawn by the package "pheatmap" in R, and the overlapping DEGs were determined by the package "Venn diagrams" in R.

\section{Enrichment Analysis}

Enrichment analysis of Gene Ontology (GO) terms and the Kyoto Encyclopedia of Genes and Genomes (KEGG) pathway for DEGs were performed by R language loaded with packages "ggplot2", "enrichplot", and "clusterProfiler". The GO analysis showed the feature of DEGs in the biological process, cellular component, and molecular function and the KEGG analysis revealed the enrichment of DEGs in the signaling pathways. $P$ and $q<0.05$ were considered statistically significant.

\section{PPI Network and Cox Regression Analysis}

The PPI network of DEGs was constructed based on the STRING database. Nodes with the confidence of interactive relationship $>0.7$ were regarded as the threshold. Subsequently, CytoHubba, which is a plug-in of Cytoscape software, was utilized to identify the top 20 hub genes. Cox regression analysis was utilized to 


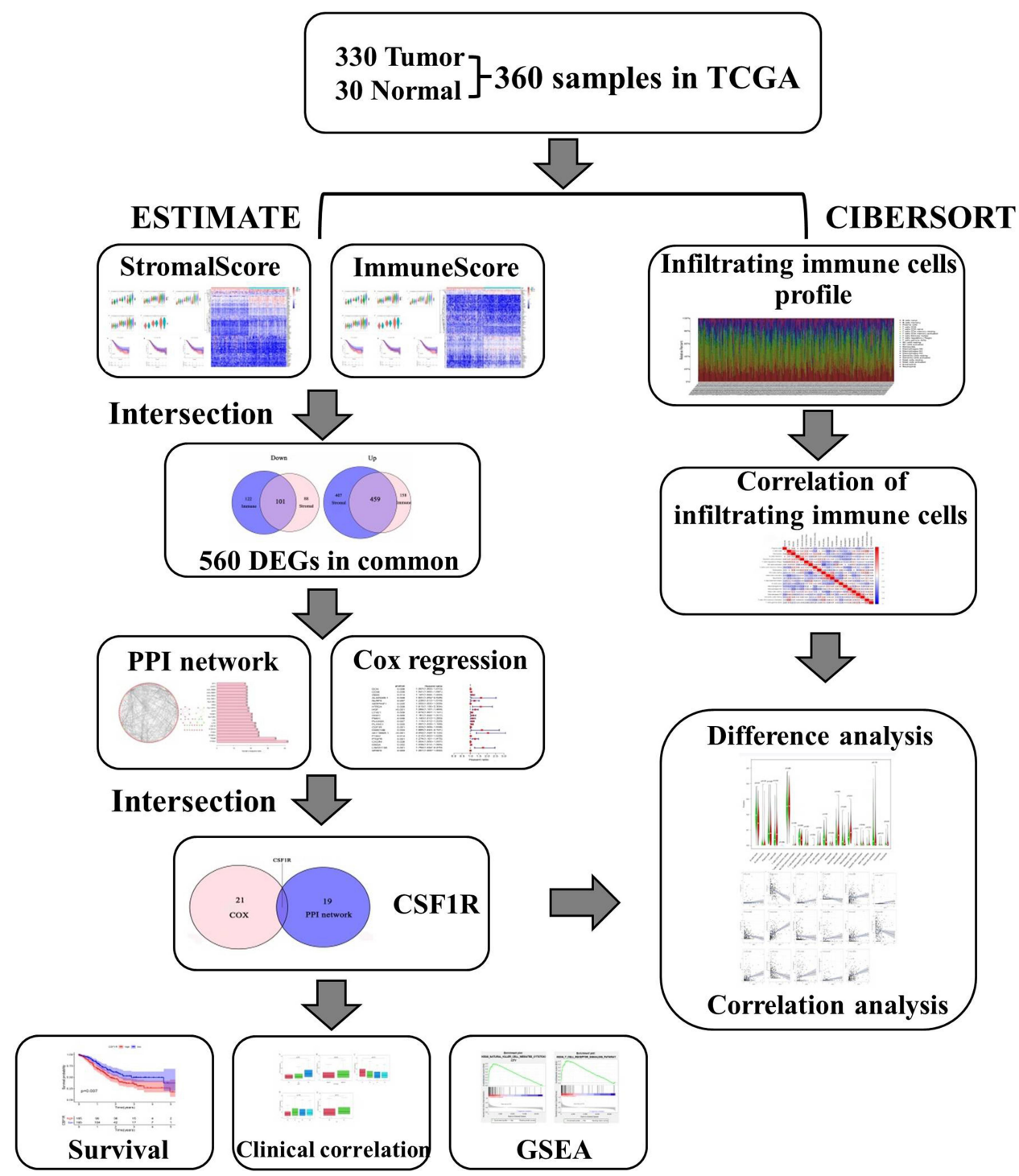

Figure I Flowchart of this study.

Abbreviations: TCGA, the Cancer Genome Atlas; DEGs, differentially expressed genes; PPI, protein-protein interaction; GSEA, gene set enrichment analysis.

determine prognostic DEGs. DEGs with $p<0.05$ for survival comparison were considered as prognostic DEGs. Venn diagrams were used to visualize the common gene CSF1R between prognostic DEGs in univariate Cox regression and the top 20 hub genes in the PPI network.

\section{Gene Set Enrichment Analysis (GSEA) and CIBERSORT Algorithm}

GC patients were classified into low-expression and highexpression groups based on CSF1R expression. To explore the role of CSF1R in regulating the GC microenvironment,
Gene set enrichment analysis for the KEGG pathway was performed using GSEA version 3.0 downloaded from Broad Institute. KEGG gene sets for enrichment analysis were obtained from the Molecular Signatures Database. NOM $\mathrm{p}<0.05$ and FDR $<0.25$ were regarded as the threshold.

Then, CIBERSORT algorithm was applied to assess the proportion of immune cells infiltrated in the TME using the LM22 signature. LM22 signature consisting of 547 genes was used to identify 22 types of infiltrating immune cells. Furthermore, we performed the difference and correlation analyses to explore the correlation between CSF1R expression and the immune cells infiltrated in the TME $(P<0.05)$. 


\section{Statistical Analysis}

Statistical analyses were performed via R software (version 4.0.2) and Perl (version 5.30.1) in this study. All statistical methods and appropriate $\mathrm{R}$ packages were described throughout the study. $P<0.05$ was set as the cutoff criterion.

\section{Results}

The Correlation of Stromal, Immune, and ESTIMATE Scores with Survival and Clinicopathological Characteristics

We downloaded gene expression information and clinical data of $330 \mathrm{GC}$ cases from TCGA. All the patients diagnosed as adenomas or adenocarcinomas were included in our study. The clinicopathological characteristics of GC patients are listed in Table 1. Subsequently, we calculated the stromal, immune, and ESTIMATE scores of GC samples by the ESTIMATE algorithm. The stromal and immune scores ranged from -726.16 to 941.62 and -287.49 to 1418.40 , respectively. The ESTIMATE scores, which are the combination of immune and stromal scores, were distributed between -788.22 to 2118.38 .

We compared the stromal/immune/ESTIMATE scores among the GC patients under grade, TNM classification, and stage. A significant correlation was observed between the above scores and grade, stage, and $\mathrm{T}$ classification. However, all scores were not associated with $\mathrm{N}$ classification and $\mathrm{M}$ classification (Figure 2). Furthermore, to evaluate the correlation between the stromal/immune/ESTIMATE scores and survival of GC patients, we divided them into the lowscore and high-score groups and compared the overall survival rate in the two groups. Intriguingly, the survival curves revealed that the stromal high-score group had a lower survival rate than the low-score group. Patients with high immune/ESTIMATE scores had a lower survival rate than those with low scores, although no statistical differences were found (Figure 3).

\section{DEGs Identification and Enrichment}

\section{Analysis}

We performed the differential analysis to determine TME-related DEGs. Heatmaps showed the differential gene expression profiles according to the stromal and
Table I Clinicopathological Characteristics of GC Patients from TCGA Database

\begin{tabular}{|c|c|}
\hline $\begin{array}{l}\text { Clinicopathological } \\
\text { Characteristics }\end{array}$ & $\begin{array}{c}\text { Number of Gastric Cancer } \\
\text { Patients }(\mathrm{N}=330)\end{array}$ \\
\hline \multicolumn{2}{|l|}{ Age (years) } \\
\hline$\leq 60$ & $105(31.8 \%)$ \\
\hline$>60$ & $224(67.9 \%)$ \\
\hline Unknown & I (0.3\%) \\
\hline \multicolumn{2}{|l|}{ Gender } \\
\hline Male & 207 (62.7\%) \\
\hline Female & $123(37.3 \%)$ \\
\hline \multicolumn{2}{|l|}{ Grade } \\
\hline GI & $8(2.4 \%)$ \\
\hline $\mathrm{G} 2$ & $122(37.0 \%)$ \\
\hline G3 & 193 (58.5\%) \\
\hline Unknown & 7 (2.1\%) \\
\hline \multicolumn{2}{|l|}{ Stage } \\
\hline Stage I & 44 (I3.3\%) \\
\hline Stage II & 101 (30.6\%) \\
\hline Stage III & $13 \mid(39.7 \%)$ \\
\hline Stage IV & 32 (9.7\%) \\
\hline Unknown & $22(6.7 \%)$ \\
\hline \multicolumn{2}{|l|}{$\mathrm{T}$ classification } \\
\hline TI & I 8 (5.5\%) \\
\hline $\mathrm{T} 2$ & 67 (20.3\%) \\
\hline T3 & 153 (46.4\%) \\
\hline $\mathrm{T} 4$ & $84(25.5 \%)$ \\
\hline Unknown & $8(2.4 \%)$ \\
\hline \multicolumn{2}{|l|}{$N$ classification } \\
\hline No & $96(29.1 \%)$ \\
\hline NI & $86(26.1 \%)$ \\
\hline N2 & 68 (20.6\%) \\
\hline N3 & 64 (19.4\%) \\
\hline Unknown & $16(4.8 \%)$ \\
\hline \multicolumn{2}{|l|}{ M classification } \\
\hline Mo & 294 (89.1\%) \\
\hline MI & 22 (6.7\%) \\
\hline Unknown & 14 (4.2\%) \\
\hline
\end{tabular}

Abbreviation: GC, gastric cancer.

immune scores (Figure 4A and B). A total of 1055 DEGs were identified between the stromal low-score and high-score groups. Among them, 866 genes were significantly upregulated, while the other 189 genes were downregulated $\left(\left|\log _{2} \mathrm{FC}\right|>1\right.$ and FDR $\left.<0.05\right)$. Simultaneously, 840 DEGs were obtained from the immune score groups, including 617 upregulated genes and 223 downregulated genes $\left(\left|\log _{2} \mathrm{FC}\right|>1\right.$ and 

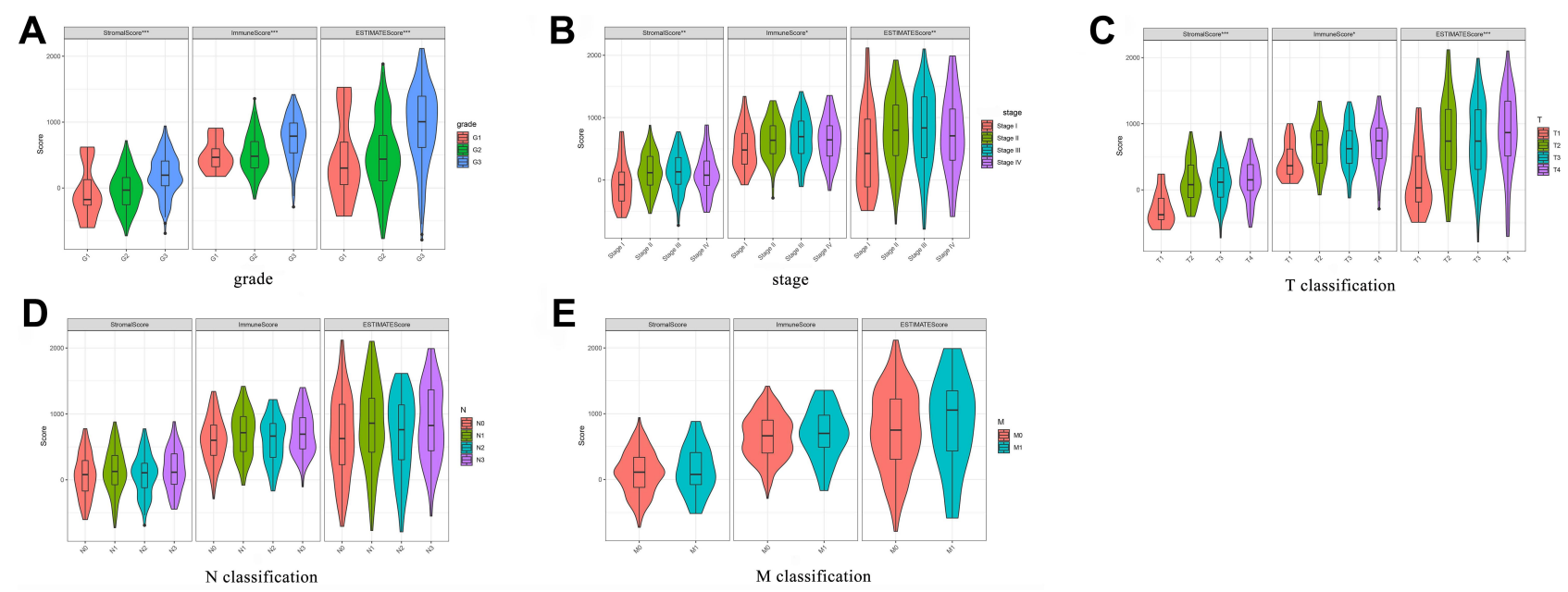

Figure 2 The correlation of stromal, immune, and ESTIMATE scores with clinicopathological characteristics (A-E).
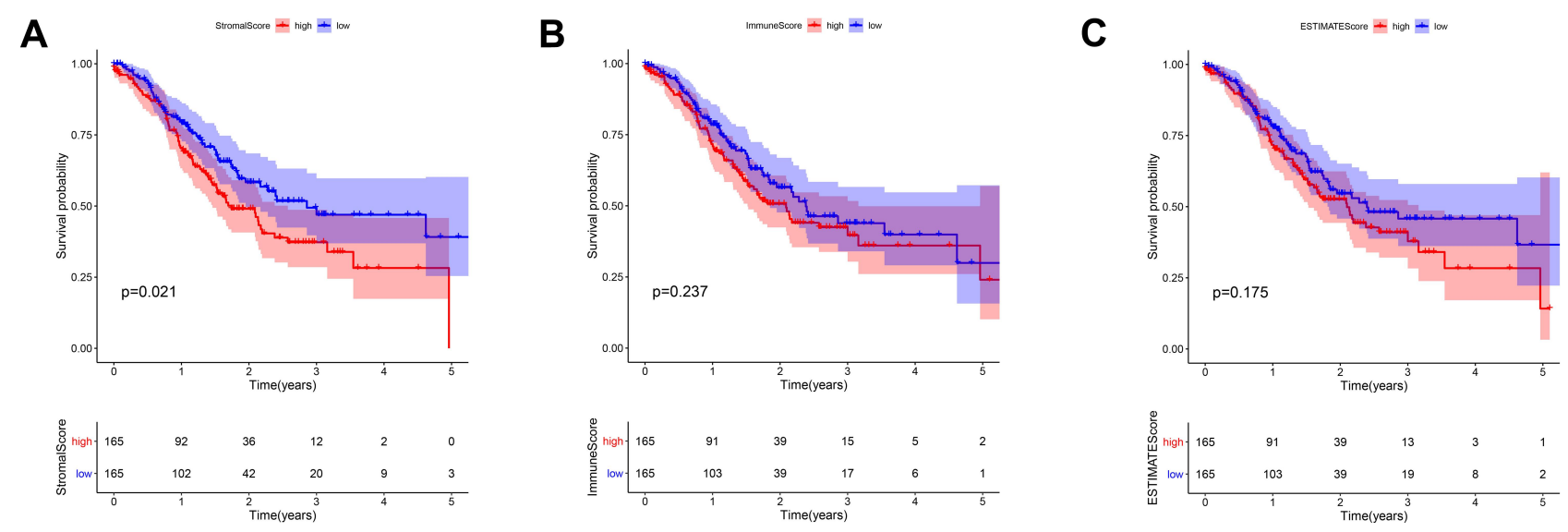

Figure 3 The correlation of stromal, immune, and ESTIMATE scores with the survival of GC patients (A-C). Abbreviation: GC, gastric cancer.

FDR $<0.05$ ). Finally, 560 DEGs in both the stromal and immune score groups were identified as TME-related DEGs (Figure 4C and D).

Furthermore, we performed functional enrichment analysis on 560 TME-related DEGs to identify their primary functions. GO enrichment analysis showed that DEGs were significantly enriched in the immunerelated terms, including regulation of immune effector process, immune response-activating signal transduction, and immune response-activating cell surface receptor signaling pathway (Figure 5A). The KEGG enrichment analysis also indicated that most of the DEG-related pathways were correlated with immune response (Figure 5B).

\section{PPI Network and Cox Regression Analysis}

The PPI network was constructed through the STRING database to analyze the interaction of DEGs (Figure 6A). Using the CytoHubba plug-in, we identified the top 20 hub genes by calculating each node's connection degree in the network (Figure 6B). Univariate Cox regression analysis for GC patients' survival was conducted to identify 22 prognostic DEGs (Figure 6C). Furthermore, the intersection analysis between the top 20 hub genes in the PPI network and 22 prognostic DEGs was carried out, and CSF1R was found to be the most critical gene (Figure 6D). 


\section{A}

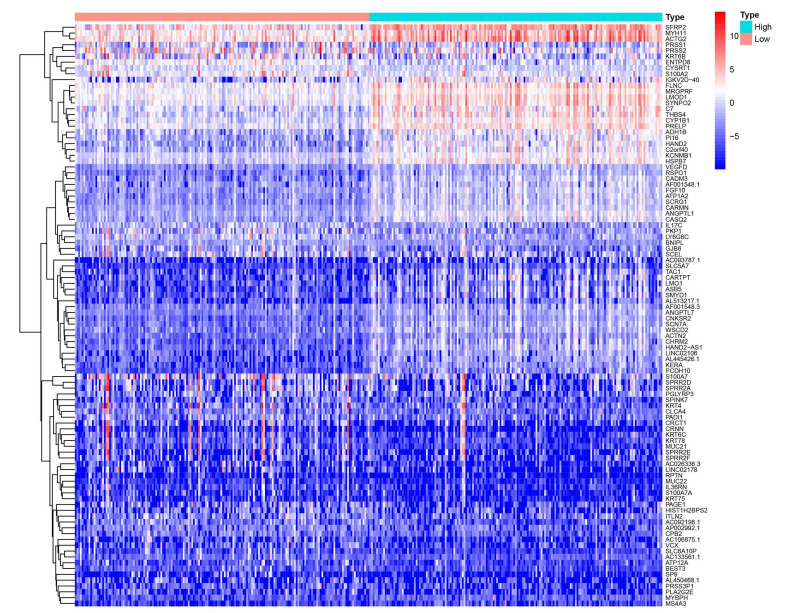

C

Down

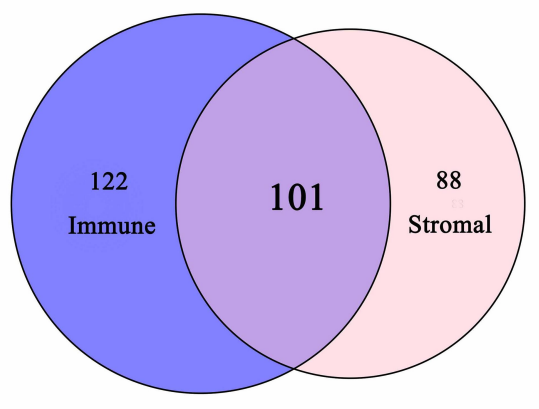

B

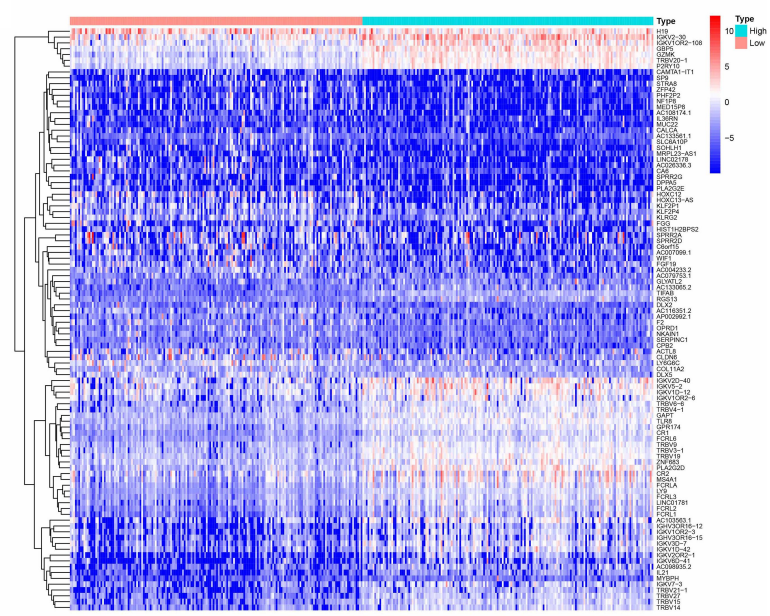

D

Up

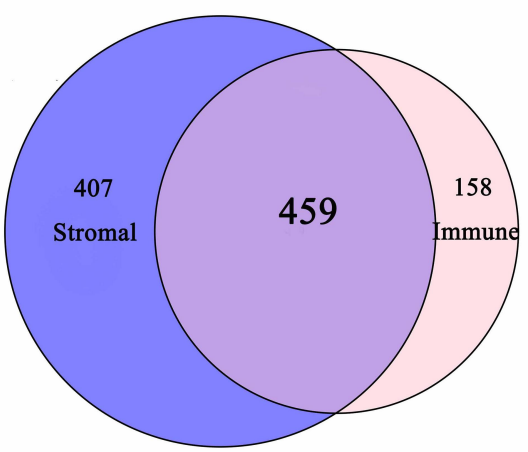

Figure 4 Gene expression profiles of GC patients with different stromal and immune scores. (A) Heatmap of DEGs between the stromal high-score and low-score groups. (B) Heatmap of DEGs between the immune high-score and low-score groups. (C) Venn diagram of commonly downregulated DEGs in the stromal and immune score groups. (D) Venn diagram of commonly upregulated DEGs in the stromal and immune score groups.

Abbreviations: GC, gastric cancer; DEGs, differentially expressed genes.

A

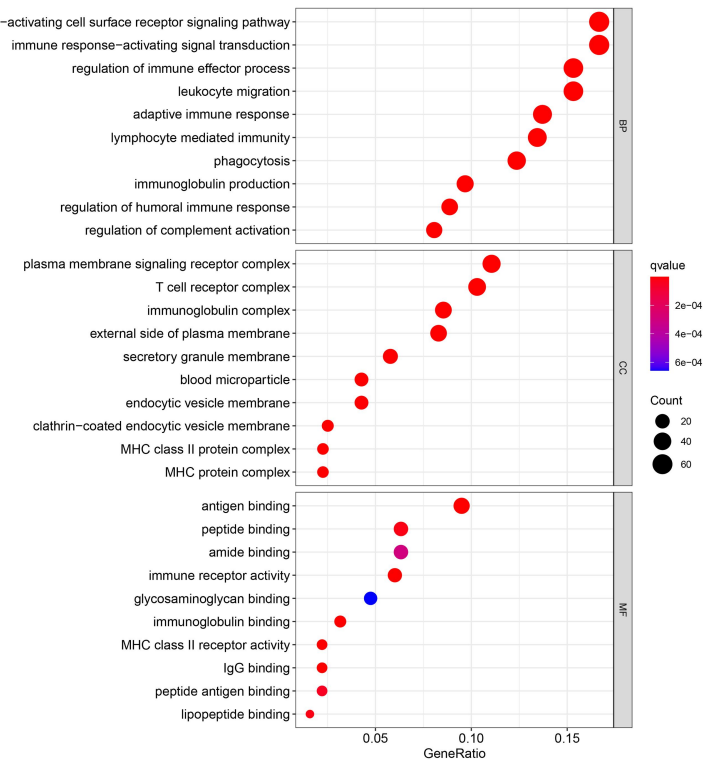

B

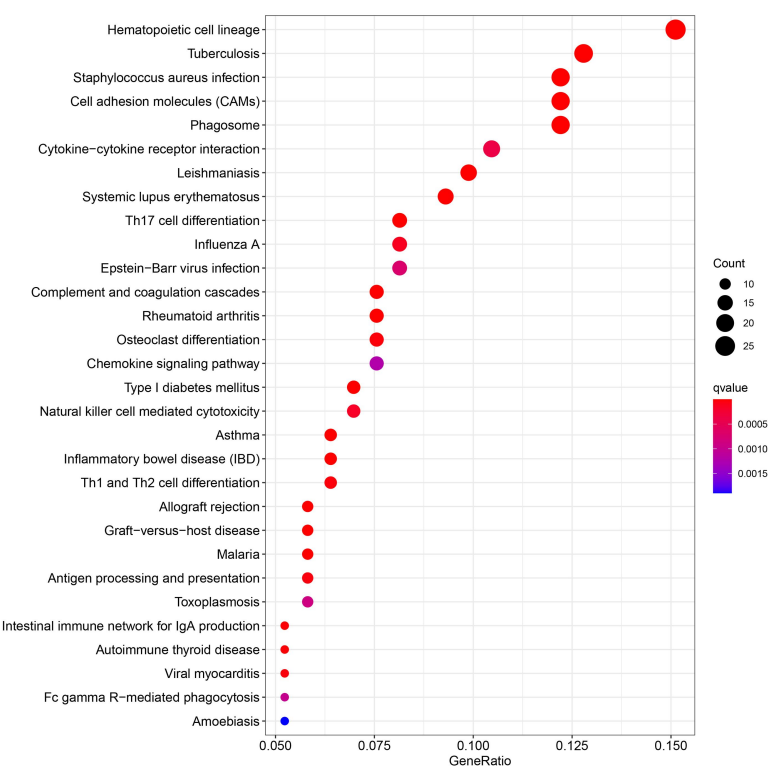

Figure 5 Functional enrichment analysis of 560 DEGs. (A) GO enrichment analysis of DEGs. (B) KEGG enrichment analysis of DEGs. Abbreviations: DEGs, differentially expressed genes; GO, Gene Ontology; KEGG, Kyoto Encyclopedia of Genes and Genomes. 
A

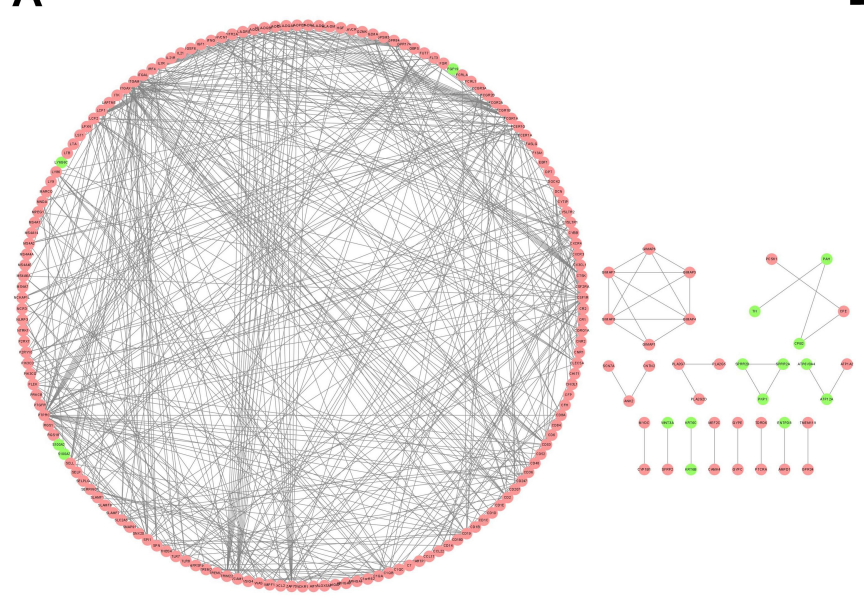

C

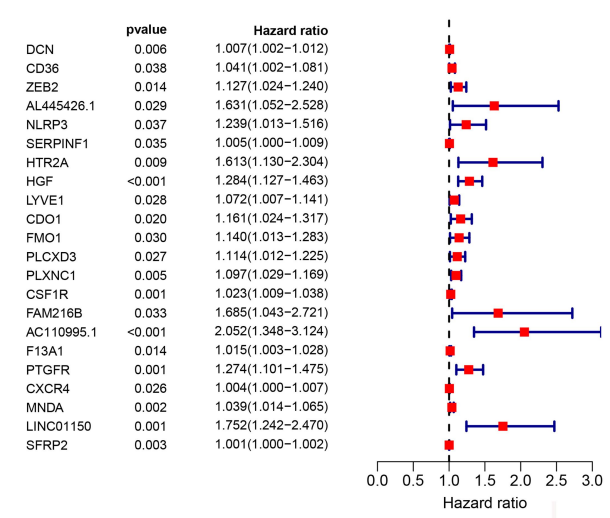

B

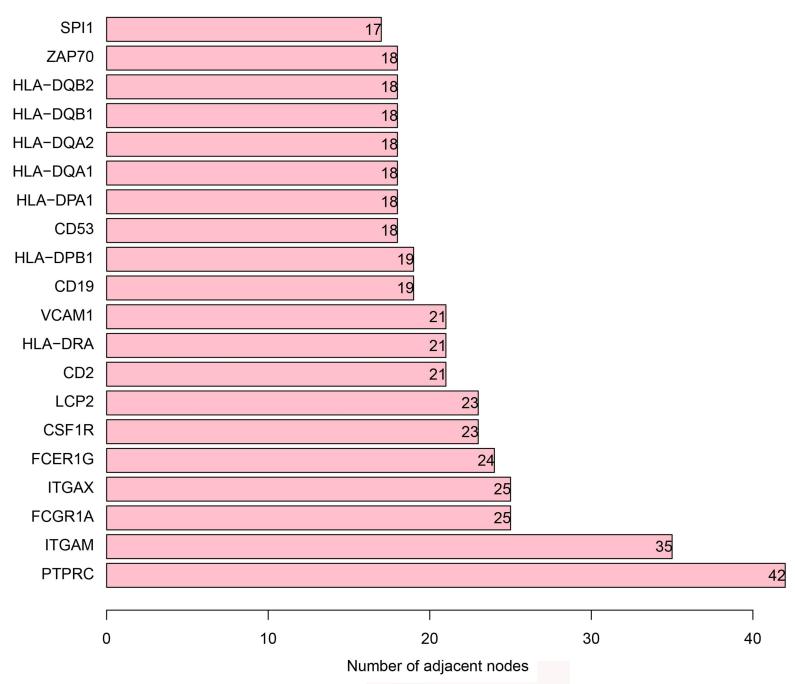

D

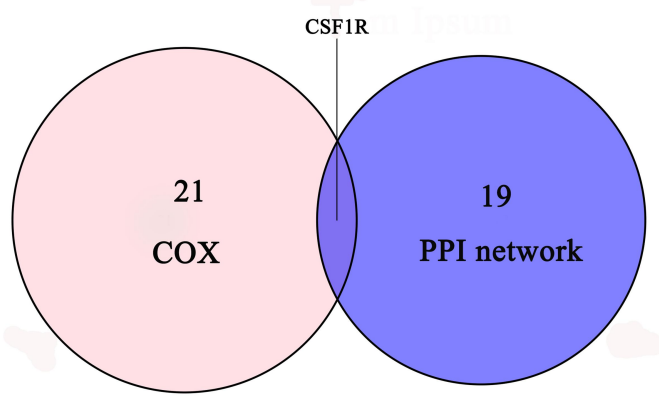

Figure 6 PPI network and univariate Cox regression analysis. (A) PPI network of 560 DEGs. (B) The top 20 genes ordered by the number of nodes in PPI network. (C) Forest plot of univariate Cox regression analysis(D) Venn diagram showing the common DEGs shared by the top 20 genes in PPI network and prognostic genes in Cox. Abbreviations: DEGs, differentially expressed genes; PPI, protein-protein interaction.

\section{The Correlation of the Survival and Clinicopathological Characteristics with CSFIR Expression}

To evaluate the role of CSF1R in the GC patients' survival, we classified all samples into low-expression and high-expression groups based on CSF1R expression. The Kaplan-Meier survival curve revealed that GC patients with CSF1R low expression had a better overall survival rate than those with CSF1R high expression (Figure 7). Furthermore, we evaluated the relationship between the expression of CSF1R and clinicopathological parameters. The result revealed that CSF1R expression was associated with grade and stage (Figure 8A and B). However, there were no significant differences in CSF1R expression among the $\mathrm{GC}$ patients with $\mathrm{T}$ classification, $\mathrm{N}$ classification and $\mathrm{M}$ classification (Figure $8 \mathrm{D}$ and $\mathrm{E}$ ).

\section{GSEA}

To explore the role of CSF1R in regulating the GC microenvironment, GSEA was performed based on the expression of CSF1R. We found that CSF1R was involved in immune-related signaling pathways, including $\mathrm{T}$ cell receptor signaling pathway and natural killer cell mediated cytotoxicity (Figure 9). The results implied that CSF1R might take part in the modulation of immune-active status in the GC microenvironment.

\section{The Correlation of the Fraction of Infiltrating Immune Cells with CSFIR Expression}

The fraction of immune cells infiltrated in the TME was analyzed through the CIBERSORT algorithm (Figure 10). Then, the difference and correlation analyses were performed 


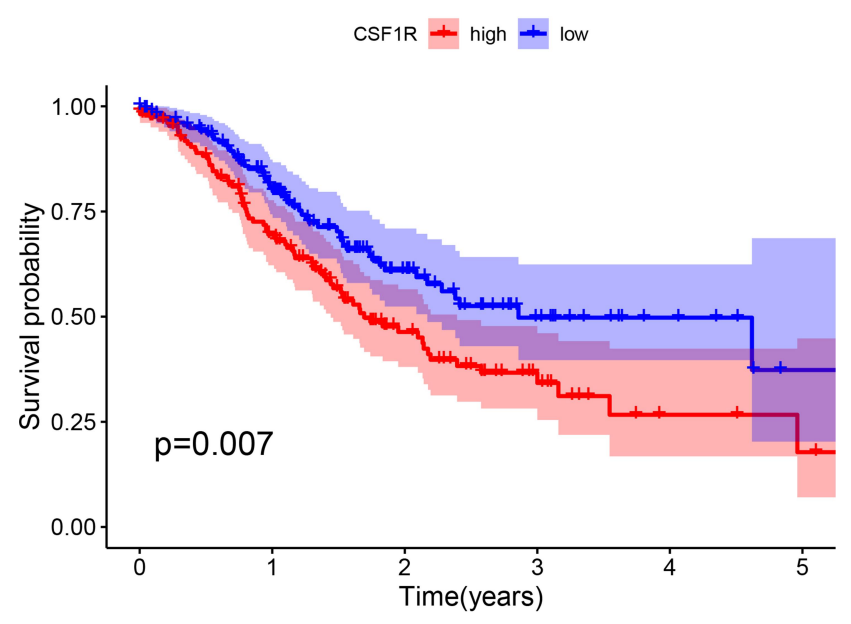

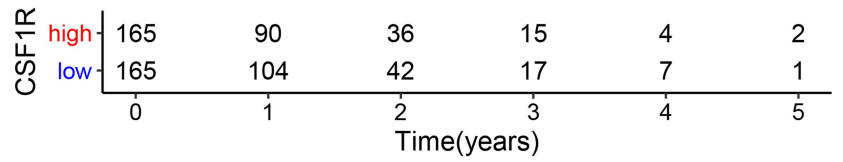

Figure 7 The correlation of CSFIR expression with the survival of GC patients. Abbreviation: GC, gastric cancer.

to explore the correlation between the immune cells infiltrated in the TME and CSF1R expression. The results indicated that a total of 14 kinds of infiltrating immune cells were related to the expression of CSF1R (Figure 11).

\section{Discussion}

During the past decades, advances in high-throughput sequencing techniques and bioinformatics have improved our understanding of transcriptional alterations in GC. More and more biomarkers have been identified to be correlated with the diagnosis and prognosis of GC. However, the crucial regulators in the GC microenvironment and the change of the TME in GC progression have not been elucidated. In this study, the stromal, immune, and ESTIMATE scores were significantly related to the clinicopathological parameters of $\mathrm{GC}$, such as grade, stage, and $\mathrm{T}$ classification. The results revealed that stromal and immune cells infiltrated in the TME might play a crucial role in GC progression. To explore the underlying mechanisms of TME change, we identified TME-related DEGs and performed enrichment analysis, construction of the PPI network, and COX regression analysis. GO and KEGG enrichment analysis indicated that most DEGs were remarkably involved in immune responses. Importantly, CSF1R was also found to be related to the clinicopathological characteristics (stage and grade) and the survival of GC patients. The results implied that CSF1R might play an essential role in modulating the TME and could be a promising therapeutic target and prognostic marker of GC.

CSF1R is a member of the type III protein tyrosine kinase receptor family and plays a central role in many diseases, such as chronic inflammatory diseases, bone disorders, and tumors. ${ }^{23}$ CSF1R can be activated either in a paracrine manner or in an autocrine manner, then
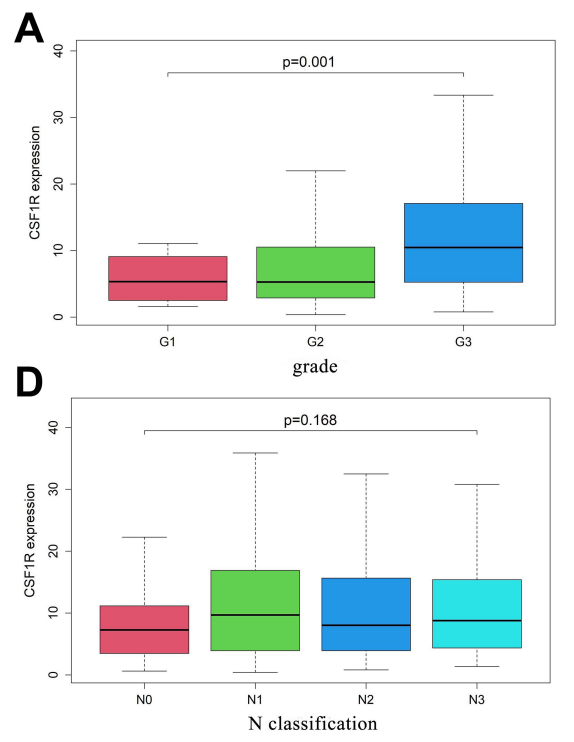
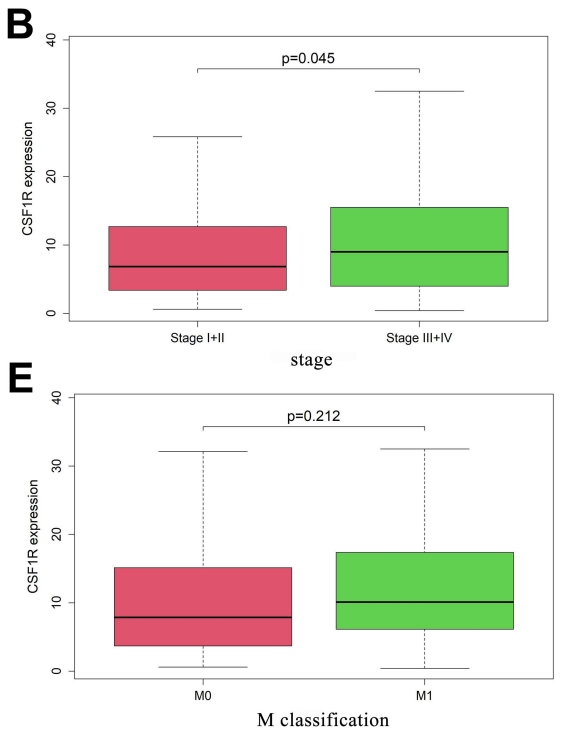

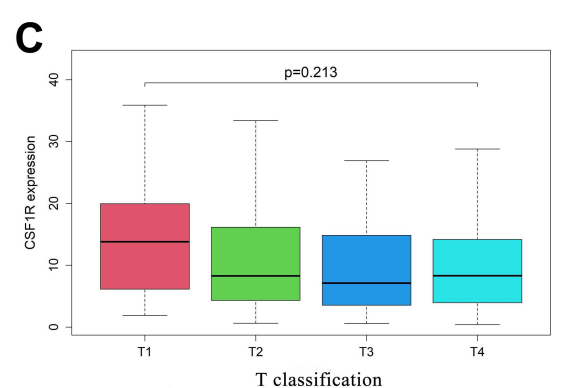

T classification

Figure 8 The correlation of CSFIR expression with clinicopathological characteristics of GC patients (A-E). Abbreviation: GC, gastric cancer. 


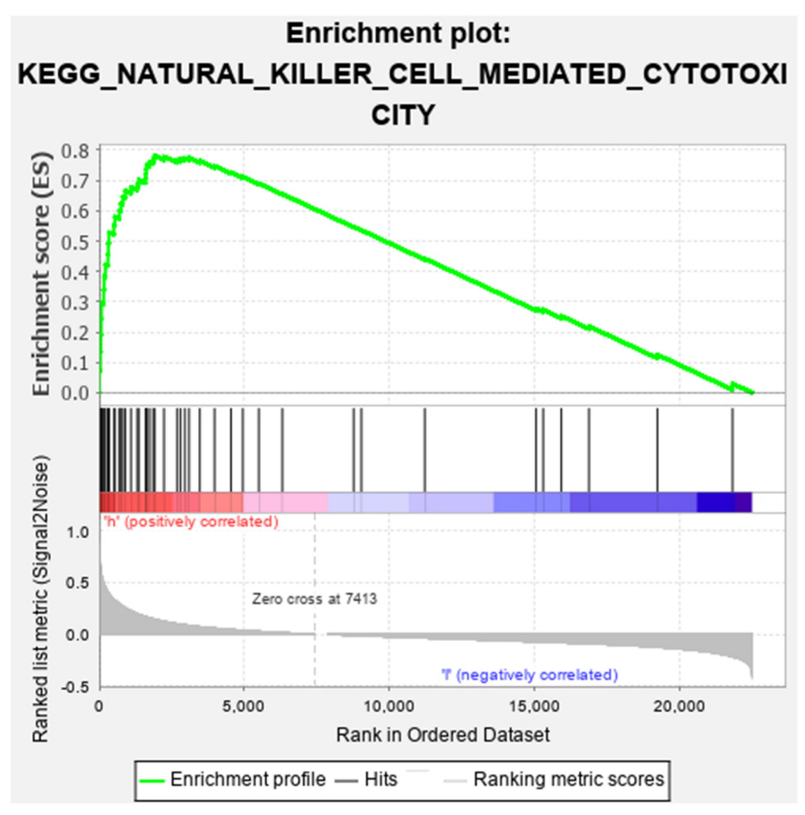

Figure 9 Gene set enrichment analysis of CSFIR.

enhancing the progression and metastasis of tumors. $^{24,25}$ For example, the expression of CSF1R and CSF1 in neoplastic epithelial cells was predictive for ipsilateral recurrence and significantly correlated with the survival of breast cancer patients. ${ }^{26,27}$ In addition, CSF1R was found to be upregulated in clear cell renal cell carcinoma, and its expression was positively associated with TNM stage, metastasis, and poor survival. $^{28}$ The elevated expression of CSF1R also was reported in Hodgkin/Reed-Sternberg (HRS) cell lines, and CSF1R signaling was demonstrated to be necessary for the growth of HRS cells. ${ }^{29}$ Recently, the upregulated expression of CSF1R or CSF1 was greatly correlated with the progression and prognosis of patients with GC. Moreover, high coexpression of CSF1R and CSF1 was also an independent prognostic factor for overall survival. ${ }^{30}$ Our results also showed that CSF1R was significantly related to the development and survival of GC patients. These findings further implied that CSF1R might be a potential biomarker for GC diagnosis and prognosis.

Furthermore, we further explored the potential role of CSF1R in regulating the TME. GSEA showed that CSF1R participated in immune-related pathways. The results implied that $\mathrm{CSF} 1 \mathrm{R}$ might take part in the

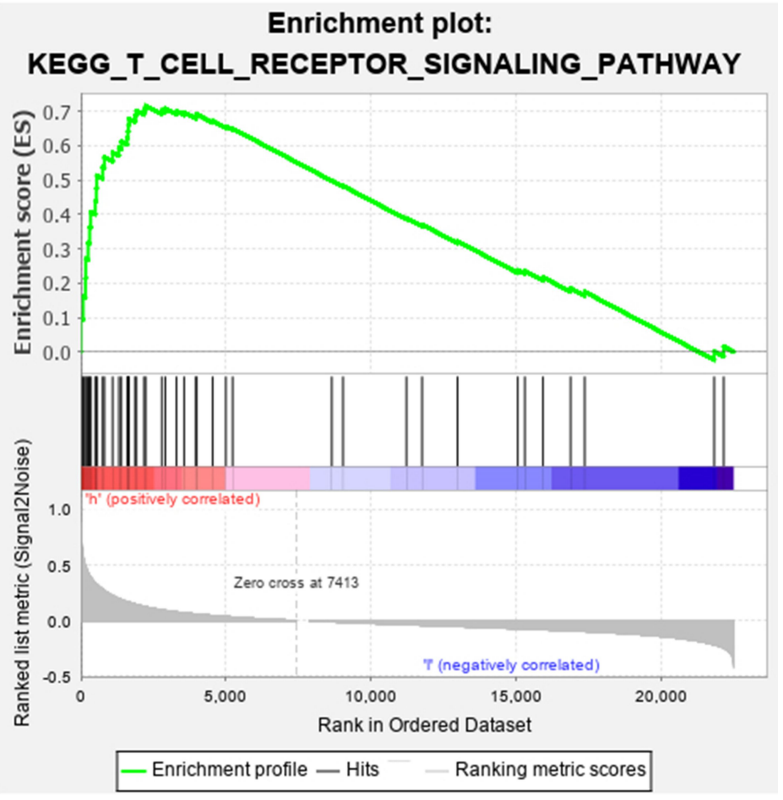

regulation of immune-active status in the GC microenvironment. Then, using the CIBERSORT algorithm, we estimated the proportion of immune cells infiltrated in the TME. The difference and correlation analyses were performed to explore the correlation between the expression of CSF1R and the immune cells infiltrated in the TME. The results revealed that the expression of CSF1R correlated with several kinds of infiltrating immune cells, including tumor-associated macrophages (TAMs), NK cells, B cells, Neutrophils, Eosinophils, $\mathrm{T}$ cells, Dendritic cells, and so on. Recently, several studies have indicated that inhibition of CSF1R can modulate both the number and the function of TAMs in different types of cancer. ${ }^{31-35}$ For example, Mitchem et al revealed that targeting TAMs by inhibiting CSF1R decreased the numbers of infiltrating immune cells, relieved immunosuppression, and improved chemotherapeutic efficacy of pancreatic ductal adenocarcinoma. ${ }^{31}$ Pyonteck et al showed that an inhibitor of CSF1R was utilized to target TAMs in a mouse model of proneural glioblastoma multiforme, which significantly regressed established tumors and increased survival. ${ }^{33}$ Furthermore, a recent study revealed that CSF1R inhibition decreased the abundance of TAMs within cervical tumors and the associated stroma and delayed the 


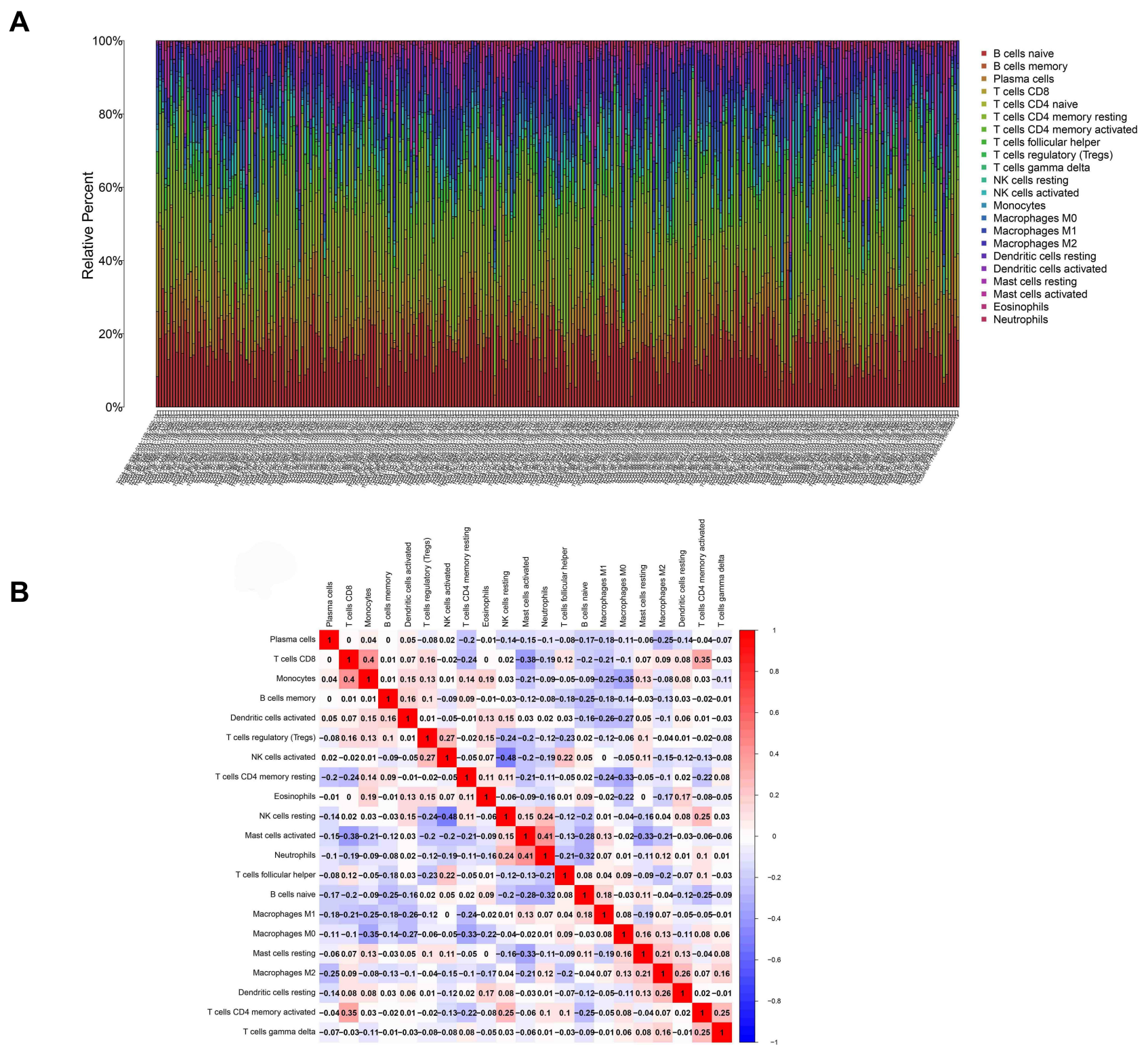

Figure 10 Infiltrating immune cells profile in gastric cancer samples and correlation analysis. (A) The proportion of 22 kinds of infiltrating immune cells. (B) The correlation between 22 kinds of infiltrating immune cells.

growth of established neoplasms. ${ }^{35}$ In addition to TAMs, CSF1R can also be detected on other cells within the TME, including neutrophils, dendritic cells, and myeloid-derived suppressor cells. ${ }^{36-38}$ All data suggested that CSF1R might be a promising crucial regulator for the microenvironment of $\mathrm{GC}$ and participate in regulating immune-active status in TME. However, further studies are needed to identify the exact immune cell atlas in the GC patients' microenvironment with different CSF1R expression levels.

\section{Conclusion}

In summary, we applied the ESTIMATE algorithm to estimate the stromal, immune, and ESTIMATE scores. Then, we concluded that stromal and immune cells infiltrated in the TME might play a crucial role in GC progression. We further explored the TME-related DEGs and found CSF1R associated with GC development and survival. More interestingly, CSF1R might participate in regulating immune-active status in TME and could be a promising biomarker for GC therapy 


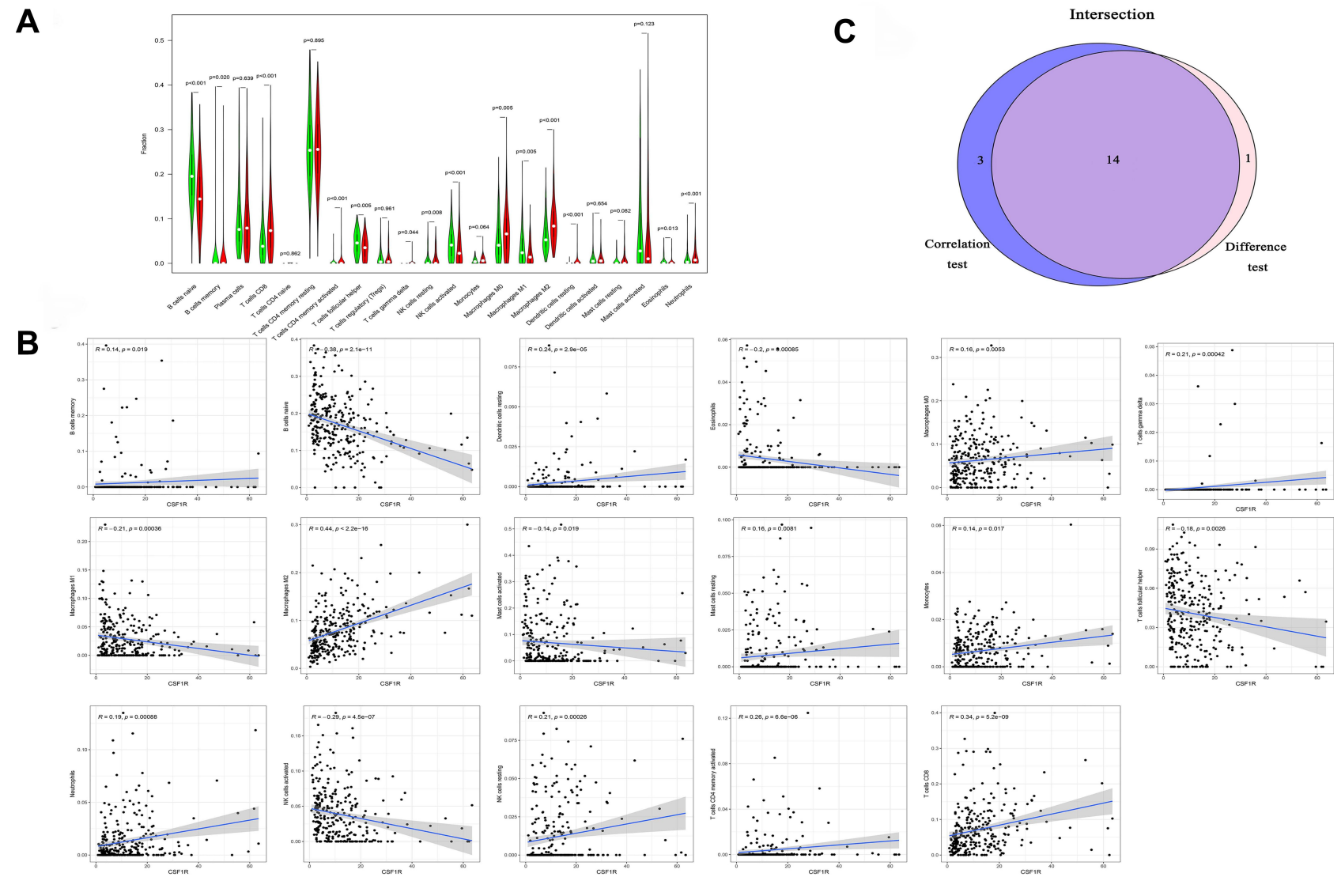

Figure II The association between the proportion of infiltrating immune cells and the expression of CSFIR. (A) Violin plot showed the ratio differentiation of infiltrating immune cells with the expression of CSFIR. (B) Scatter plot showed the correlation of infiltrating immune cells proportion with CSFIR expression. (C) Venn plot displayed 14 kinds of infiltrating immune cells associated with CSFIR expression by difference and correlation analysis.

and prognosis. However, more experiments are needed to clarify the potential mechanisms of CSF1R in the microenvironment of GC patients.

\section{Acknowledgments}

This study was supported by the National Key Research and Development Program of China (2018YFC2002000). We thank all those participants in this study.

\section{Author Contributions}

All authors made substantial contributions to conception and design, acquisition of data, or analysis and interpretation of data; took part in drafting the article or revising it critically for important intellectual content; agreed to submit to the current journal; gave final approval of the version to be published; and agree to be accountable for all aspects of the work.

\section{Disclosure}

The authors report no conflicts of interest in this work.

\section{References}

1. Bray F, Ferlay J, Soerjomataram I, Siegel RL, Torre LA, Jemal A. Global cancer statistics 2018: GLOBOCAN estimates of incidence and mortality worldwide for 36 cancers in 185 countries. CA Cancer J Clin. 2018;68(6):394-424. doi:10.3322/caac.21492

2. Rugge M, Genta RM, Di Mario F, et al. Gastric cancer as preventable disease. Clin Gastroenterol H. 2017;15(12):1833-1843. doi:10.1016/j. cgh.2017.05.023

3. Smyth EC, Nilsson M, Grabsch HI, van Grieken NCT, Lordick F. Gastric cancer. Lancet. 2020;396(10251):635-648. doi:10.1016/ S0140-6736(20)31288-5

4. Zeng D, Li M, Zhou R, et al. Tumor microenvironment characterization in gastric cancer identifies prognostic and immunotherapeutically relevant gene signatures. Cancer Immunol Res. 2019;7(5):737-750. doi:10.1158/2326-6066.CIR-18-0436

5. Binnewies M, Roberts EW, Kersten K, et al. Understanding the tumor immune microenvironment (TIME) for effective therapy. Nat Med. 2018;24(5):541-550. doi:10.1038/s41591-018-0014-x

6. Pasini FS, Zilberstein B, Snitcovsky I, et al. A gene expression profile related to immune dampening in the tumor microenvironment is associated with poor prognosis in gastric adenocarcinoma. $J$ Gastroenterol. 2014;49(11):1453-1466. doi:10.1007/s00535-0130904-0

7. Singh SR, Rameshwar P, Siegel P. Targeting tumor microenvironment in cancer therapy. Cancer Lett. 2016;380(1):203-204. doi:10.1016/j. canlet.2016.04.009 
8. Valkenburg KC, de Groot AE, Pienta KJ. Targeting the tumour stroma to improve cancer therapy. Nat Rev Clin Oncol. 2018;15 (6):366-381.

9. Chen Q, Liu G, Liu S, et al. Remodeling the tumor microenvironment with emerging nanotherapeutics. Trends Pharmacol Sci. 2018;39 (1):59-74. doi:10.1016/j.tips.2017.10.009

10. Jiang Y, Zhang Q, Hu Y, et al. ImmunoScore signature: a prognostic and predictive tool in gastric cancer. Ann Surg. 2018;267(3):504-513. doi:10.1097/SLA.0000000000002116

11. Thompson ED, Zahurak M, Murphy A, et al. Patterns of PD-L1 expression and CD8 $\mathrm{T}$ cell infiltration in gastric adenocarcinomas and associated immune stroma. Gut. 2017;66(5):794-801. doi:10.1136/gutjnl-2015-310839

12. Zeng D, Zhou R, Yu Y, et al. Gene expression profiles for a prognostic immunoscore in gastric cancer. Br J Surg. 2018;105 (10):1338-1348. doi:10.1002/bjs.10871

13. Chen F, Zhuang X, Lin L, et al. New horizons in tumor microenvironment biology: challenges and opportunities. BMC Med. 2015;13:45. doi:10.1186/s12916-015-0278-7

14. He KY, Ge D, He MM. Big data analytics for genomic medicine. Int J Mol Sci. 2017;18(2):412. doi:10.3390/ijms18020412

15. Yoshihara K, Shahmoradgoli M, Martinez E, et al. Inferring tumour purity and stromal and immune cell admixture from expression data. Nat Commun. 2013;4:2612. doi:10.1038/ncomms3612

16. Jia D, Li S, Li D, Xue H, Yang D, Liu Y. Mining TCGA database for genes of prognostic value in glioblastoma microenvironment. Aging. 2018;10(4):592-605. doi:10.18632/aging.101415

17. Xu WH, Xu Y, Wang J, et al. Prognostic value and immune infiltration of novel signatures in clear cell renal cell carcinoma microenvironment. Aging. 2019;11(17):6999-7020. doi:10.18632/ aging. 102233

18. Alonso MH, Ausso S, Lopez-Doriga A, et al. Comprehensive analysis of copy number aberrations in microsatellite stable colon cancer in view of stromal component. Br J Cancer. 2017;117(3):421-431. doi:10.1038/bjc.2017.208

19. Newman AM, Liu CL, Green MR, et al. Robust enumeration of cell subsets from tissue expression profiles. Nat Methods. 2015;12 (5):453-457. doi:10.1038/nmeth.3337

20. Bense RD, Sotiriou C, Piccart-Gebhart MJ, et al. Relevance of tumor-infiltrating immune cell composition and functionality for disease outcome in breast cancer. J Natl Cancer Inst. 2017;109(1): djw192. doi:10.1093/jnci/djw192

21. Chen B, Khodadoust MS, Liu CL, Newman AM, Alizadeh AA. Profiling tumor infiltrating immune cells with CIBERSORT. Methods Mol Biol. 2018;1711:243-259.

22. Washington K. 7th edition of the AJCC cancer staging manual: stomach. Ann Surg Oncol. 2010;17(12):3077-3079. doi:10.1245/ s10434-010-1362-z

23. El-Gamal MI, Al-Ameen SK, Al-Koumi DM, Hamad MG, Jalal NA, $\mathrm{Oh} \mathrm{CH}$. Recent advances of colony-stimulating factor-1 receptor (CSF-1R) kinase and its inhibitors. J Med Chem. 2018;61 (13):5450-5466. doi:10.1021/acs.jmedchem.7b00873

24. Stanley ER, Chitu V. CSF-1 receptor signaling in myeloid cells. Cold Spring Harb Perspect Biol. 2014;6(6):a021857. doi:10.1101/ cshperspect.a021857
25. Achkova D, Maher J. Role of the colony-stimulating factor (CSF)/ CSF-1 receptor axis in cancer. Biochem Soc Trans. 2016;44 (2):333-341. doi:10.1042/BST20150245

26. Kluger HM, Dolled-Filhart M, Rodov S, Kacinski BM, Camp RL, Rimm DL. Macrophage colony-stimulating factor-1 receptor expression is associated with poor outcome in breast cancer by large cohort tissue microarray analysis. Clin Cancer Res. 2004;10(1 Pt 1):173-177. doi:10.1158/1078-0432.CCR-0699-3

27. Maher MG, Sapi E, Turner B, et al. Prognostic significance of colony-stimulating factor receptor expression in ipsilateral breast cancer recurrence. Clin Cancer Res. 1998;4(8):1851-1856.

28. Yang L, Liu Y, An H, et al. High expression of colony-stimulating factor 1 receptor associates with unfavorable cancer-specific survival of patients with clear cell renal cell carcinoma. Ann Surg Oncol. 2016;23(3):1044-1052. doi:10.1245/s10434-015-4911-7

29. Lamprecht B, Walter K, Kreher S, et al. Derepression of an endogenous long terminal repeat activates the CSF1R proto-oncogene in human lymphoma. Nat Med. 2010;16(5):571-579, 571p following 579. doi:10.1038/nm.2129

30. Okugawa Y, Toiyama Y, Ichikawa $\mathrm{T}$, et al. Colony-stimulating factor-1 and colony-stimulating factor-1 receptor co-expression is associated with disease progression in gastric cancer. Int $J$ Oncol. 2018;53(2):737-749. doi:10.3892/ijo.2018.4406

31. Mitchem JB, Brennan DJ, Knolhoff BL, et al. Targeting tumor-infiltrating macrophages decreases tumor-initiating cells, relieves immunosuppression, and improves chemotherapeutic responses. Cancer Res. 2013;73(3):1128-1141. doi:10.1158/00085472.CAN-12-2731

32. Mok S, Koya RC, Tsui C, et al. Inhibition of CSF-1 receptor improves the antitumor efficacy of adoptive cell transfer immunotherapy. Cancer Res. 2014;74(1):153-161. doi:10.1158/ 0008-5472.CAN-13-1816

33. Pyonteck SM, Akkari L, Schuhmacher AJ, et al. CSF-1R inhibition alters macrophage polarization and blocks glioma progression. Nat Med. 2013;19(10):1264-1272. doi:10.1038/nm.3337

34. Priceman SJ, Sung JL, Shaposhnik Z, et al. Targeting distinct tumor-infiltrating myeloid cells by inhibiting CSF-1 receptor: combating tumor evasion of antiangiogenic therapy. Blood. 2010;115 (7):1461-1471. doi:10.1182/blood-2009-08-237412

35. Strachan DC, Ruffell B, Oei Y, et al. CSF1R inhibition delays cervical and mammary tumor growth in murine models by attenuating the turnover of tumor-associated macrophages and enhancing infiltration by $\mathrm{CD} 8(+) \mathrm{T}$ cells. Oncoimmunology. 2013;2(12): e26968. doi:10.4161/onci.26968

36. Cannarile MA, Weisser M, Jacob W, Jegg AM, Ries CH, Ruttinger D. Colony-stimulating factor 1 receptor (CSF1R) inhibitors in cancer therapy. J Immunother Cancer. 2017;5(1):53. doi:10.1186/ s40425-017-0257-y

37. Pedersen MB, Danielsen AV, Hamilton-Dutoit SJ, et al. High intratumoral macrophage content is an adverse prognostic feature in anaplastic large cell lymphoma. Histopathology. 2014;65 (4):490-500. doi:10.1111/his.12407

38. DeNardo DG, Brennan DJ, Rexhepaj E, et al. Leukocyte complexity predicts breast cancer survival and functionally regulates response to chemotherapy. Cancer Discov. 2011;1(1):54-67. doi:10.1158/21598274.CD-10-0028 


\section{Publish your work in this journal}

Pharmacogenomics and Personalized Medicine is an international, peer-reviewed, open access journal characterizing the influence of genotype on pharmacology leading to the development of personalized treatment programs and individualized drug selection for improved safety, efficacy and sustainability. This journal is indexed on the American Chemical Society's Chemical Abstracts Service (CAS). The manuscript management system is completely online and includes a very quick and fair peer-review system, which is all easy to use. Visit http://www.dovepress.com/testimonials.php to read real quotes from published authors. 